\title{
Preface
}

\section{Advances in Surgical Critical \\ Care 2017: Growing Population and Personalized Protocols}

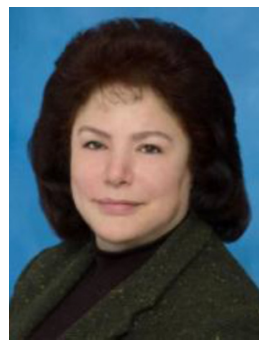

Lena M. Napolitano, MD, FACS, FCCP, MCCM

Editor

Critical care is a young specialty, initiated in 1953 when poliomyelitis patients required invasive mechanical ventilation, and even earlier for surgical patients recovering from anesthesia or traumatic injuries. ${ }^{1,2}$ The burden of critical illness globally is high and has been increasing as our population ages. ${ }^{3}$

Paradoxically, critical care continues to grow in a shrinking hospital system. Although hospital beds decreased by $2.2 \%$ from 2000 to 2010 in the United States, critical care beds increased by $17.8 \%$, with a $20.4 \%$ increase in the critical care medicine-to-hospital bed ratio (13.5\%-16.2\%). During this same time period, critical care costs nearly doubled (92.2\%; \$56-\$108 billion) and the proportion of critical care cost to the gross domestic product increased by $32.1 \% .^{4}$

The provision of critical care globally is more variable, with a documented remarkable degree of variation in both the number of adult intensive care unit (ICU) beds (7-fold difference from least to greatest) and the volume of ICU admissions (10-fold difference) between seven different countries, but with a high inverse correlation between the number of ICU beds and hospital mortality for ICU patients. ${ }^{5}$

Use of critical care services for surgical patients is also widely variable, and significant variations in mortality in surgical critically ill patients have been reported. ${ }^{6-8}$ Management of critically ill patients is challenging, and the field of critical care is undergoing rapid change. The population of critically ill patients is highly heterogeneous, with different comorbidities and varying types and degrees of organ dysfunction.

So how best can we improve the survival of critically ill patients in the context of these significant challenges? Intensivists must be knowledgeable about recent advances and randomized trials in the critically ill, ${ }^{9}$ but must also be capable of providing personalized medicine to identify which patients can potentially benefit from new interventions that have been rigorously tested. ${ }^{10}$ 
This issue of Critical Care Clinics documents important progress made in the field of critical care and provides an update of the significant evidence-based advances in critical care that result in improved patient outcomes. In this issue, leading experts provide succinct reviews of important critical care advances in the broad areas of multidisciplinary patient care bundles, delirium, early mobility, airway, respiratory, renal, sepsis, transfusion, and nutrition support. They review cutting-edge advances and provide evidence-based recommendations for best practices in critical care.

I extend my deepest gratitude to our authors for their excellent contributions to this important issue on Advances in Critical Care, and to Richard W. Carlson, Patrick J. Manley, and Casey Potter and the entire editorial team.

We hope that this issue of Critical Care Clinics will assist in timely dissemination of critical care knowledge by providing the updated evidence base to ensure optimal care to all of our critically ill patients.

Lena M. Napolitano, MD, FACS, FCCP, MCCM Division of Acute Care Surgery [Trauma, Burns, Surgical Critical Care, Emergency Surgery] Department of Surgery University of Michigan Health System Room 1C340A University Hospital 1500 East Medical Center Drive Ann Arbor, MI 48109-5033, USA

E-mail address: lenan@umich.edu

\section{REFERENCES}

1. Ibsen B. The anaesthetist's viewpoint on the treatment of respiratory complications in poliomyelitis during the epidemic in Copenhagen, 1952. Proc R Soc Med 1954;47:72-4.

2. Grenvik A, Pinsky MR. Evolution of the intensive care unit as a clinical center and critical care medicine as a discipline. Crit Care Clin 2009;25:239-50.

3. Adhikari NK, Fowler RA, Bhagwanjee S, et al. Critical care and the global burden of critical illness in adults. Lancet 2010;376(9749):1339-46.

4. Halpern NA, Goldman DA, Tan KS, et al. Trends in critical care beds and use among population groups and Medicare and Medicaid beneficiaries in the United States: 2000-2010. Crit Care Med 2016;44(8):1490-9.

5. Wunsch $\mathrm{H}$, Angus DC, Harrison DA, et al. Variation in critical care services across North America and Western Europe. Crit Care Med 2008;36:2787-93.

6. Pearse RM, Moreno RP, Bauer P, et al, European Surgical Outcomes Study (EuSOS) group for the trials groups of the European Society of Intensive Care Medicine and the European Society of Anaesthesiology. Mortality after surgery in Europe: a 7 day cohort study. Lancet 2012;380(9847):1059-65.

7. International Surgical Outcomes Study Group. Global patient outcomes after elective surgery: prospective cohort study in 27 low-, middle- and high-income countries. Br J Anaesth 2016;117(5):601-9.

8. Gillies MA, Harrison EM, Pearse RM, et al. Intensive care utilization and outcomes after high-risk surgery in Scotland: a population-based cohort study. Br J Anaesth 2017;118(1):123-31. 
9. Bellomo R, Landoni G, Young P. Improved survival in critically ill patients: are large RCTs more useful than personalized medicine? Yes. Intensive Care Med 2016;42(11):1775-7.

10. Vincent JL. Improved survival in critically ill patients: are large RCTs more useful than personalized medicine? No. Intensive Care Med 2016;42(11):1778-80. 